\title{
Distortion of meteor count rates due to cosmic radio noise and atmospheric particularities
}

\author{
G. Stober ${ }^{1, *}$, C. Jacobi ${ }^{1}$, and D. Keuer ${ }^{2}$ \\ ${ }^{1}$ University Leipzig, Institute for Meteorology, Stephanstr. 3, 04103 Leipzig, Germany \\ ${ }^{2}$ University of Rostock, Leibniz-Institute of Atmospheric Physics, Schlossstr. 6, 18225 Kühlungsborn, Germany \\ * present address: University of Rostock, Leibniz-Institute of Atmospheric Physics, Schlossstr. 6, \\ 18225 Kühlungsborn, Germany
}

\begin{abstract}
The determination of the meteoroid flux is still a scientifically challenging task. This paper focusses on the impact of extraterrestrial noise sources as well as atmospheric phenomena on the observation of specular meteor echoes. The effect of cosmic radio noise on the meteor detection process is estimated by computing the relative difference between radio loud and radio quiet areas and comparing the monthly averaged meteor flux for fixed signal-to-noise ratios or fixed electron line density measurements. Related to the cosmic radio noise is the influence of D-layer absorption or interference with sporadic E-layers, which can lead to apparent day-to-day variation of the meteor flux of $15-20 \%$.
\end{abstract}

\section{Introduction}

During the last years all-sky SKiYMET meteor radars have become a frequently used sensor system to study the mesopause region. The diversity of radar experiments reaches from mesopause region wind (Jacobi et al., 2007, 2008) and temperature (Hocking et al., 1999, 2001; Singer et al., 2004; Stober et al., 2008) measurements to astrophysical observations on meteor fluxes (Campbell-Brown and Close, 2007), radiant position (Jones and Jones, 2006) or velocity determination (Baggaley, 2003; Hocking et al., 2004; Stober, 2009) of single meteors.

Recently, this kind of meteor radar is also used to estimate the meteoroid masses (Stober et al., 2009). The basis for this analysis is the calibration of the radar, which was performed according to Latteck et al. (2008). A calibrated radar enables one to determine the electron line density $q$ in the specular

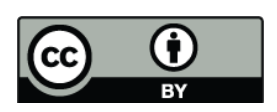

Correspondence to: G. Stober (stober@iap-kborn.de) point, which is a direct measure of the ablation rate $d m / d t$ of the meteoroid:

$q=\frac{\beta(v)}{\mu v^{2}} \frac{d m}{d t}$.

Here $\beta$ is the ionization efficiency, $\mu$ is the mass of an ablated atom and $v$ is the velocity of the meteor. In combination with meteor ablation models and the determined meteor flux it is possible to estimate the deposited meteoric mass in the upper mesosphere and lower thermosphere (MLT) region.

The determination of the total meteor flux is a scientifically challenging task for two reasons. There is no sensor available to observe the complete spectra of meteoric particles reaching from faint radio meteors $(\mu \mathrm{m}$-sized) to the optical bright fireballs/bolides (m-sized). The other aspect is that each method suffers from measurement limitations e.g. observation geometry or detection thresholds. The meteor radar measurements provide a meteor count rate according to the radar parameters (antenna gain, frequency, noise level, observation geometry). This meteor count rate can, in principle, be converted to a real meteor flux by considering these parameters. In particular, the observation geometry for specular meteors requires some computational effort. CampbellBrown and Jones (2006) estimated the meteor flux for the six sporadic meteor sources considering the minimum detectable electron line density, the declination angle of the source radiant and the population index for sporadic meteoroids.

The minimum detectable electron line density is given by the background radio noise level, which defines the detection threshold for a meteor and also the minimum measurable meteoroid mass (Stober et al., 2009). The upper detection threshold is given by the requirement that only partial reflecting meteor trails (underdense meteors) can be used to determine the electron line density. If total reflection occurs

Published by Copernicus Publications on behalf of the URSI Landesausschuss in der Bundesrepublik Deutschland e.V. 


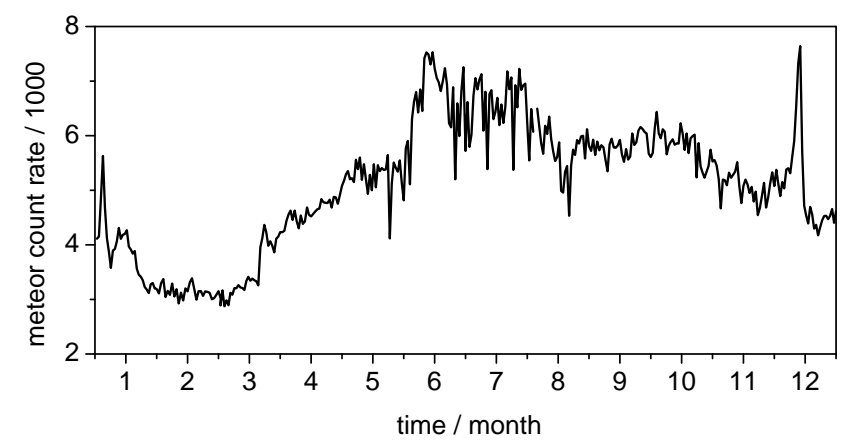

Fig. 1. August 2004 to October 2009 composite of the daily meteor count rate over Collm.

on the trail the mass ablation rate cannot be measured, due to a saturation of electron line density (overdense meteors).

Estimating the meteor flux from meteor radar observations of specular echoes therefore includes only meteors within the mass range given by the minimum and maximum detectable electron line density or meteoroid mass, respectively. However, the meteor flux can be extrapolated by using the population index $r$ for given meteor population. This index describes the magnitude distribution or mass distribution, respectively, within a specific group of meteors with a power law. The sporadic meteor background can be characterized with a population index of $r=2$. This parameter enables one to estimate the expected meteor count rate outside the detectable mass range and is therefore a suitable parameter to estimate the total meteor flux within a meteor population independent of selection effects due to the choice of radar parameters.

\section{Collm meteor radar}

The measurements presented in this paper were recorded with the Collm meteor radar $\left(51.3^{\circ} \mathrm{N}, 13^{\circ} \mathrm{E}\right)$. The radar is a commercially produced all-sky SKiYMET radar, which detects ambipolar plasma trails of meteoroids entering the Earth's atmosphere. The radar measures the backscattered radio wave at the specular point of the rapidly diffusing trails. The interferometric design of the receiving antennas provides the position of the meteor in the sky. The radial drift velocity, measured through the receiver phase shift with time at each receiver, is suitable to observe MLT winds. The diffusion of the meteor trails can be used to estimate the mesopause region temperature through estimating the ambipolar diffusion coefficient. A detailed description of the system can be found in Hocking, 1999; Hocking et al., 2001, 2004.

The receiving antenna array consists of five two-element Yagis, which are aligned in an asymmetric cross with base lengths between the receivers of 2 and 2.5 wavelengths. The transmitter antenna is placed a few meters aside the receiver antenna array. The transmitter antenna is a three-element
Table 1. Technical data and main parameters employed by the Collm meteor radar.

\begin{tabular}{lc}
\hline geographic position & $51.3^{\circ} \mathrm{N}, 13^{\circ} \mathrm{E}$ \\
frequency & $36.2 \mathrm{MHz}$ \\
pulse repetition frequency & $2144 \mathrm{~Hz}$ \\
transmitting power & $6 \mathrm{~kW}$ \\
height range & $70-110 \mathrm{~km}$ \\
range resolution & $2 \mathrm{~km}$ \\
coherent integrations & $4-$ point \\
pulse width & $13 \mu \mathrm{s}$ \\
angular resolution & $2^{\circ}$ \\
receiver bandwidth & $50 \mathrm{kHz}$ \\
transmitting antenna & $3-$ element Yagi \\
receiving antenna & 5-channel interfero- \\
& meter of 2-element Yagi \\
\hline
\end{tabular}

Yagi. This leads to a characteristic dipole antenna pattern (Stober et al., 2009). Each antenna is connected to a receiver with a $70 \mathrm{~m}$ coaxial cable. The cable loss introduced through these cables is $1.61 \mathrm{~dB}$. In Table 1 the radar settings and major parameters are summarized.

For each confirmed meteor event, $4 \mathrm{~s}$ of data are recorded, one second before the $t_{0}$-point of the meteor (defined as the time when the meteor head reaches specular condition) and $3 s$ after it (Hocking et al., 2001). The sky temperature is derived using only the first 400 pulses, i.e. before the $t_{0}$-point, of the in-phase and quadrature components to avoid any contamination from the meteor trail. A detailed description of the procedure can be found in Stober et al., 2009.

\section{Meteor climatology}

The meteor flux as measured by standard meteor radars is characterized by a higher meteor count rate during the summer and autumn months and a clear minimum in spring. This seasonal pattern is mainly caused by the different sporadic meteor sources (Campbell-Brown and Jones, 2006; Stober, 2009). However, a more detailed analysis of the meteor flux indicates a strong variability from day to day, which cannot be explained by the different meteor sources or the occurrence of meteor showers. This variability can, among others, be caused by external interferences, ionospheric D-layers or sporadic E-layers. In Fig. 1 the measured meteor flux over Collm is shown. The figure clearly indicates the position of the prominent meteor showers of the Quadrantids (begin of January) and the Geminids (mid December), as well as the general increased meteor flux during the summer months. However, the graph also shows that there is a strong variability during the summer, with day-to-day differences of up to 1000 meteors. In the next section we investigate probable reasons for these variations. 


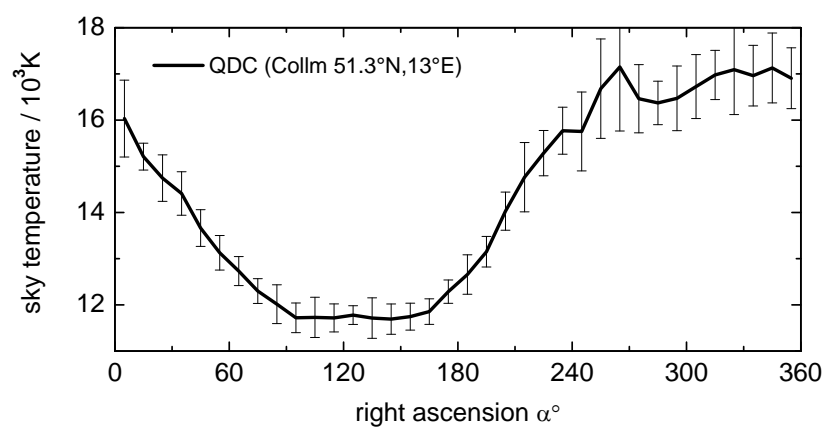

Fig. 2. QDC used for the determination of the absorption.

\section{Background radio noise}

Besides the variation of the background radio noise due to existing D-layers or sporadic E-layers, there is a natural variation of the radio signature due to the beam pointing direction. For wide beam meteor radars, this variation is mainly caused by our home galaxy. In Fig. 2 the resulting Quiet Day Curve (QDC) of the Collm meteor radar is shown. The procedure to determine this QDC is described in detail by Stober et al., 2009b. The figure illustrates the characteristic sidereal variation of the cosmic radio noise for a beam moving along the celestial sphere.

The variation during one sidereal day is in the order of 1.8$2 \mathrm{~dB}$ for the Collm meteor radar. This results in the fact that the meteor detection threshold shifts by approximately $2 \mathrm{~dB}$ to smaller meteoroids during times, when the beam points towards a region with a reduced cosmic radio intensity and vice versa. In Fig. 3 this effect is demonstrated by comparing the meteor count rate for a constant signal-to-noise ratio and a constant electron line density as detection threshold. The two curves represent one month averages of the daily meteor count rate over local time. The radar applies a constant trigger level of $5 \mathrm{~dB}$ above the noise floor, which represents a compromise between a high acceptance rate and noise spike rejections. However, for a precise estimate of the meteor flux the detectable mass range has to be kept constant and should not include any variation with time. The differences between the two lines in Fig. 3 result from the shift in the detection threshold caused by the sidereal variation of the cosmic noise level.

The impact of the QDC ( $2 \mathrm{~dB}$ variation) on meteor count rates is approximately $8 \%$ or $200-300$ meteors per day for a constant threshold of $8 \mathrm{~dB}$. Assuming a threshold of only $6 \mathrm{~dB}$ would lead to a difference of $18 \%$ in the meteor count rate. The lower the signal-to-noise ratio, the smaller meteoroids can be detected. Hence, the estimation of the meteor flux can be significantly improved by using only meteors within a fixed electron line density interval, instead of counting all possible detections. The minimum detectable electron line density was taken as $q=6.3 \cdot 10^{12} 1 / \mathrm{m}$ to com-

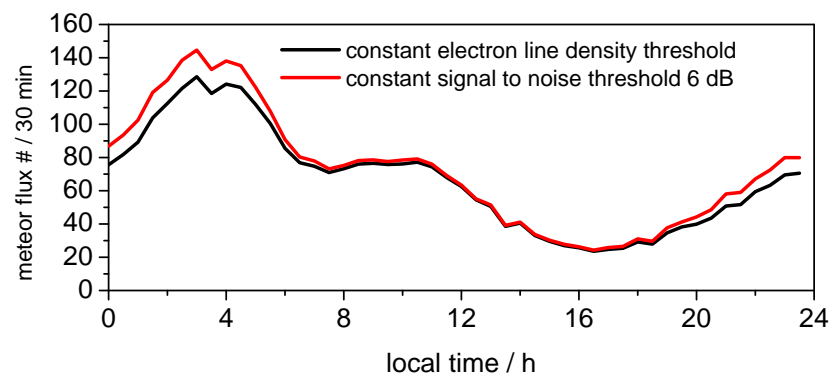

Fig. 3. Comparison of the impact of constant signal-to-noise-ratio and fixed electron line density filters on the determination of the meteor flux.

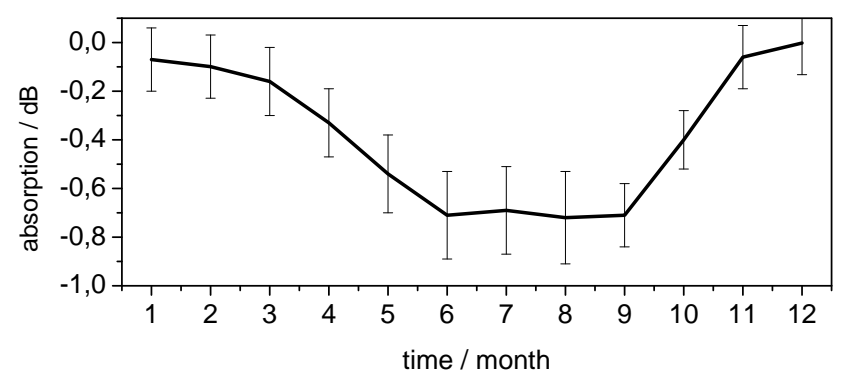

Fig. 4. Seasonal absorption measured with the Collm meteor radar.

pute the reference meteor flux. This effect is also strongly dependent on the geographic position of the meteor radar. On the southern hemisphere the QDC can reach a difference between radio loud and radio quiet areas of $8 \mathrm{~dB}$.

\section{Ionospheric distortions on the meteor count rate}

The Collm meteor radar shows strong seasonal variation of the absorption of cosmic radio noise. One reason is the location of the radar site, which is surrounded by trees. In Fig. 4 the apparent energy loss in the course of the year is visualized. The energy loss due to the surrounding vegetation was estimated to $0.5-1 \mathrm{~dB}$ by comparing the absorption curves from the meteor radar in Juliusruh with the Collm radar. The strongest absorption occurred during times with precipitation (see also Sect. 7).

The seasonal absorption consists of contributions from the radar environment and ionospheric phenomena like ionospheric D-layers or sporadic E-layers, which can be considered to explain the observed seasonal pattern. The absorption due to D-layers results in a similar effect like the diurnal variation of the cosmic radio background. The absorption was estimated by comparing a QDC with the radio noise measurements during the whole year. The result is shown in Fig. 5. The figure is based on a $4 \mathrm{~h}$ running mean absorption computed from the noise observation. 


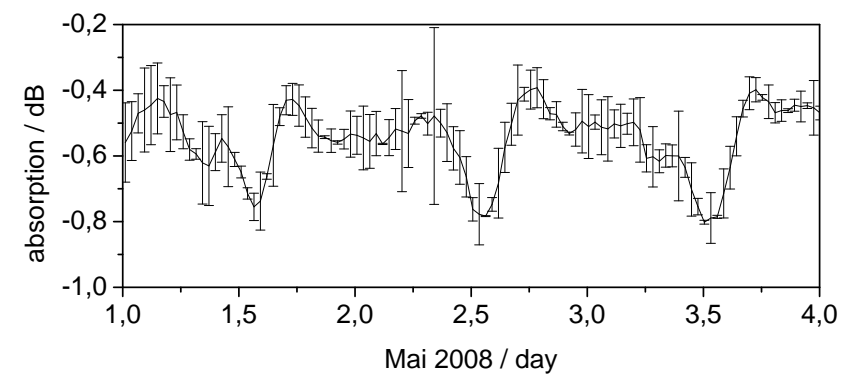

Fig. 5. Absorption computed from meteor radar noise observations.

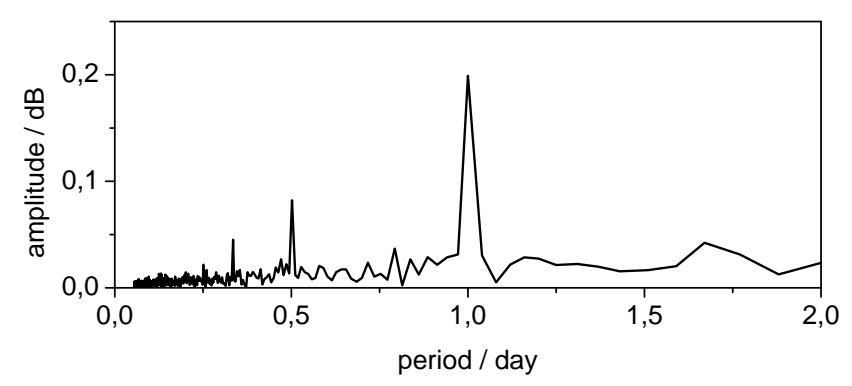

Fig. 6. Spectrum of the absorption observed in September 2008.

Figure 5 illustrates a relative absorption of $0.2-0.3 \mathrm{~dB}$ that occurs always around 12:00 LT. These values show a variation through the year with a minimum of $0.05-0.1 \mathrm{~dB}$ during the winter and the maximum of $0.3 \mathrm{~dB}$ in summer. Considering the error of the absorption measurements, which is computed to $0.2 \mathrm{~dB}$ leads to the conclusion that the absorption pattern seems not to be significant at first sight. The phase of this minimum remains stable throughout the year at 12:00 LT. A more detailed analysis reveals a minor minimum in the evening hours between 18:00-22:00 LT, which may belong to a sporadic E-layer. A Fourier transform was used to extract the characteristic frequencies from the time series. The spectral components are given in Fig. 6.

The spectrum shown in Fig. 6 indicates an apparent peak at 1 day period and further subharmonics. The calculated spectral amplitude consists of the integral absorption due to the D-layer and probable sporadic E-layers. However, the meteor radar can not deliver a constant absorption measurement due to a lot of external interferences. The derived absorption amplitude due to ionospheric phenomena is still much weaker than the absorption caused by tropospheric distortions. The seasonal course of the absorption needs to be considered in the estimation of meteor fluxes. Stober et al. (2009) demonstrated the use of a calibrated meteor radar to measure meteoroid masses from underdense trails observed in the specular point. The key element of this method is the accurate measurement of the electron line density. The seasonal absorption pattern is a particular problem for this analysis, because the radio waves have to pass the D-region

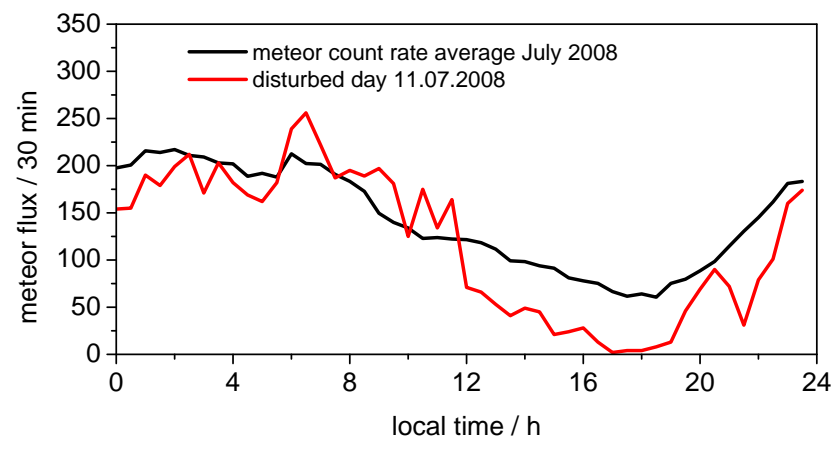

Fig. 7. Example of monthly mean meteor flux (black) compared to a disturbed day (red).

twice. The result is a signal amplitude loss of $1.4 \mathrm{~dB}$ during summer. Hence, the electron line density is biased by a factor of 2 .

\section{Tropospheric interferences}

During summer one sometimes also recognizes a decrease of the meteor count rate in the afternoon or evening hours. Figure 7 visualizes a typical situation which happened in July 2008. The black line represents the monthly averaged meteor count rate over LT. The red line visualizes the meteor count rate for 1107 2008. In Fig. 7 the meteor count rates at this day starts to decrease at 12:00 LT, which is caused by an approaching thunderstorm with extensive lightning activity. This results in an increased noise level. The radar starts to sample at a range of $6 \mathrm{~km}$, which results in strong tropospheric echoes created on the strong gradients of this thunderstorm front. The second source that may lead to an almost complete breakdown of the meteor detection are lightnings. Their plasma channels have an almost fractal structure and are therefore suitable to be a specular reflector. Also, the plasma density reaches a sufficient level to partially reflect the transmitted radio waves. However, the event on 11072008 represents an extreme heavy thunderstorm with almost $5 \mathrm{~h}$ of lightning and storm gust activity in the vicinity of the Collm radar. The storm front was also detected by the meteor radar in Juliusruh, which did record real bursts of probable meteor events in the count rate. These bursts were characterized by many signatures within a few milliseconds and covering several height gates. The typical Gaussian height distribution of the observed meteors around $90 \mathrm{~km}$ vanished during such a burst. Hence these spikes in the recorded data are caused by lightnings, which can also provide enough ionized plasma within a rapidly diffusing channel to result in measurable signal for the meteor radar. 


\section{Conclusions}

In this paper we focus on the impact of radio noise variations and ionospheric phenomena on the estimation of the meteor flux using VHF radars. The results demonstrate that the daily variation of the QDC has to be considered for the determination of the meteor flux. In the case of the Collm meteor radar the effect changes the measured count rate by $8-10 \%$. Using a fixed lower and upper electron line density threshold ensures that the measured meteor population fits into a then well defined mass range of the meteoroids.

Furthermore, we investigated the disturbances caused by ionospheric phenomena on the meteor detection process, which can be considered to be of minor importance for the meteor flux determination. However, particle precipitation or solar flares can lead to large ionospheric D-region electron line densities, which can lead to a significant absorption of the transmitted radio waves.

The biggest impact for the Collm meteor radar is the absorption caused by the properties of the radar site itself. This work points out that the electron line densities are biased towards a factor of 2 between summer and winter. This has to be considered also for the determination of corrected meteor fluxes based upon a fixed electron line density threshold. The second important distortion of the meteor flux during the summer was also identified. The event from 11072008 could be clearly related to a heavy thunderstorm crossing the Collm station.

This work represents several aspects of likely distortions of the meteor detection, which need to be corrected for to obtain reliable estimates of the meteor flux. In combination with calibrated radars a well-determined meteor flux then may be used to estimate the meteoric mass deposit in the MLT region, which is still a challenging scientific task.

Acknowledgements. Special thanks to colleagues from the IAP in Kühlungsborn for advice, useful discussions and support during the calibration. The technical support and maintenance of the radar at Collm by F. Kaiser is acknowledged. Topical Editor M. Förster thanks E. D. Schmitter, J. Wickert, and an anonymous reviewer for their help in evaluating this paper.

\section{References}

Baggaley, W.: Radar Observations, in: Meteors in the Earths Atmosphere, edited by: Williams, I. P. and Murad, E., Cambridge University Press, 123-147, 2002.

Campbell-Brown, M. and Close, S.: Meteoroid structure from radar head echoes, Mon. Not. R. Astron. Soc., 382, 1309-1316, 2007.

Campbell-Brown, M. and Jones, J.: Annual variation of sporadic radar meteor rates, Mon. Not. R. Astron. Soc., 367, 709-716, 2006.

Hocking, W.: Temperatures using radar-meteor decay times, Geophys. Res. Lett., 26, 3297-3300, 1999.

Hocking, W., Fuller, B., and Vandepeer, B.: Real-time determination of meteor-related parameters utilizing modern digital technology, J. Atmos. Solar-Terr. Phys., 63, 155-169, 2001.

Hocking, W., Singer, W., Bremer, J., Mitchell, N., Batista, P., Clemesha, B., and Donner, M.: Meteor radar temperatures at multiple sites derived with SKiYMET radars and compared to $\mathrm{OH}$, rocket and lidar measurements, J. Atmos. Solar-Terr. Phys., 66, 585-593, 2004.

Jacobi, C., Fröhlich, K., Viehweg, C., Stober, G., and Kürschner, D.: Midlatitude mesosphere/lower thermosphere meridional winds and temperatures measured with meteor radar, Adv. Space Res., 39, 1278-1283, 2007.

Jacobi, C., Stober, G., and Kürschner, D.: Connection between winter mesopause region temperatures and diurnal LF reflection height variations measured at Collm, Adv. Space Res., 41, 14281433, 2008.

Jones, J. and Jones, W.: Meteor radiant activity mapping using single-station radar observations, Mon. Not. R. Astron. Soc., 367, 1050-1056, 2006.

Latteck, R., Singer, W., Morris, R. J., Hocking, W. K., Murphy, D. J., Holdsworth, D. A., and Swarnalingam, N.: Similarities and differences in polar mesosphere summer echoes observed in the Arctic and Antarctica, Ann. Geophys., 26, 2795-2806, 2008, http://www.ann-geophys.net/26/2795/2008/.

Singer, W., von Zahn, U., and Weiß, J.: Diurnal and annual variations of meteor rates at the arctic circle, Atmos. Chem. Phys., 4, 1355-1363, 2004, http://www.atmos-chem-phys.net/4/1355/2004/.

Stober, G.: Astrophysical Studies on Meteors using a SKiYMET All-Sky Meteor Radar, Ph.D. thesis, Institute for Meteorology, University Leipzig, 2009.

Stober, G., Jacobi, C., Fröhlich, K., and Oberheide, J.: Meteor radar temperatures over Collm $\left(51.3^{\circ} \mathrm{N} 13^{\circ} \mathrm{E}\right)$, Adv. Space Res., 42, 1253-1258, 2008.

Stober, G., Singer, W., and Jacobi, C.: Meteoroid mass determination from specular observed underdense trails, J. Atmos. SolarTerr. Phys., submitted 2009.

Stober, G., Singer, W., Jacobi, C., and Hoffmann, P.: Cosmic Noise Observations using a mid-latitude Meteor Radar, J. Atmos. SolarTerr. Phys., submitted 2009. 